\title{
Course of Alcohol Symptoms and Social Anxiety Disorder from Adolescence to Young Adulthood
}

\author{
Jessica J. Black, Duncan B. Clark, Christopher S. Martin, Kevin H. Kim, Thomas J. Blaze, \\ Kasey G. Creswell, and Tammy Chung \\ Pittsburgh Adolescent Alcohol Research Center (JJB, DBC, CSM, TC), Western Psychiatric \\ Institute and Clinic, Pittsburgh, Pennsylvania; Department of Education (KHK, TJB), University of \\ Pittsburgh, Pittsburgh, Pennsylvania; and Department of Psychology (KGC), Carnegie Mellon \\ University, Pittsburgh, Pennsylvania
}

\section{Abstract}

Background-There is limited knowledge of the course of social anxiety disorder (SAD) from adolescence into adulthood, and how SAD and alcohol use disorder (AUD) symptoms change together over time. The current study examined how persistent and adolescent-limited SAD relate to alcohol symptom trajectories across adolescence and into adulthood, as well as gender differences in the course of SAD and AUD symptoms.

Methods-Participants were 788 youth (ages 12 to 18 at the baseline assessment; $46.2 \%$ female; $80.5 \%$ White) recruited from the community $(n=220)$ and from clinical programs $(n=568)$. Youth completed clinical interviews on their lifetime history of AUD symptoms and SAD at baseline and were followed through age 25 . Multivariate polynomial growth mixture modeling was used to estimate developmental trajectories for SAD and AUD symptoms separately, then together in a dual trajectory model. Gender differences were examined using a classify-analyze approach.

Results-Three SAD trajectory classes were identified: adolescent-limited (15\%), persistent (6\%), and no SAD (79\%). For AUD symptoms, 5 trajectories were identified: severe (10\%), moderate (22\%), remitting (18\%), young adult onset (22\%), and stable low (28\%). Those with a history of SAD were about twice as likely to be in the severe AUD symptom class compared to those without a history of SAD. Compared to those with persisting SAD, those in the adolescentlimited SAD class were more likely to belong to the stable low AUD trajectory. Compared to males with SAD, females with SAD were less likely to be in the moderate AUD symptom class and were more likely to be in stable low and young adult onset AUD symptom classes.

Conclusions-A history of SAD was associated with membership in the severe AUD trajectory group. The association of gender with SAD and AUD differed depending on developmental period. Future research should examine whether treating SAD in early adolescence may prevent subsequent AUD symptoms.

Copyright $@ 2015$ by the Research Society on Alcoholism.

Reprint requests: Jessica J. Black, PhD, WPIC/Pittsburgh Adolescent Alcohol Research Center, 3811 O'Hara Street, Pittsburgh, PA 15213; Tel.: 412-246-5147; Fax 412-246-6550; blackj3@upmc.edu. 


\section{Keywords}

Adolescence; Alcohol; Social Anxiety; Trajectory

Social anxiety disorder (SAD) is a common psychiatric condition that generally emerges in adolescence (Kessler et al., 2005a). SAD is characterized by excessive preoccupation with embarrassing oneself in social or performance situations, resulting in distress in or avoidance of those situations (American Psychiatric Association, 2013). In addition to SAD diagnosis, social anxiety has often been studied as a continuous variable (e.g., Norberg et al., 2009). Adolescent girls typically report greater social anxiety than adolescent boys in community samples (Van Oort et al., 2009). Regarding the course of social anxiety during adolescence, existing studies have followed youth across a relatively narrow developmental window. For example, Van Oort and colleagues (2009) followed a large community sample of adolescents over 5 years, finding that social anxiety tended to decrease from ages 10 to 12, then steadily increased from ages 12 to 15 (Van Oort et al., 2009).

Alcohol use disorder (AUD) is also a common psychiatric disorder that tends to onset during late adolescence and young adulthood (Chung and Jackson, in press; Chung and Martin, 2011). Research has characterized trajectories of adolescent alcohol use and their association with AUDs in young adulthood. For example, Chassin and colleagues (2004) conducted trajectory analyses on participants' alcohol consumption from early adolescence to young adulthood (mean ages 13.2 to 25.0) and identified 3 consumption groups: (i) heavy drinking, (ii) moderate drinking, and (iii) light drinking. Both the heavy and moderate drinking groups demonstrated an increased risk for alcohol dependence; however, only the heavy drinking group displayed persistent dependence into young adulthood rather than adolescent-limited dependence (Chassin et al., 2004).

AUD and SAD are associated with each other. AUD occurs more frequently among persons with SAD than those without SAD (Wolitzky-Taylor et al., 2012; Zimmermann et al., 2003). A national survey found that $48.2 \%$ of adults with a lifetime history of SAD had a history of AUD, compared to $29.3 \%$ of those with no history of SAD (Grant et al., 2005). Both of these disorders commonly emerge in adolescence (Grant et al., 2005; Kessler et al., 2007). However, little is known about the relationship between SAD and AUD symptoms across the entire period of adolescent development into young adulthood.

A few studies have examined the natural course of social anxiety and alcohol use during adolescence. Windle and Windle (2012) began examining youth in mid-adolescence (mean age 15.5) and found SAD was specifically related to drinking to cope in young adulthood (mean age 28.5). In a brief report on the temporal ordering of anxiety, mood, and substance use disorders, Wolitzky-Taylor and colleagues (2012) followed youth for 4 years during mid-adolescence (mean ages 16.9 to 20.9) and found that baseline SAD predicted onset of AUDs at follow-up; however, AUDs did not predict later SAD. A recent study examined social anxiety symptoms throughout early adolescence and found that higher levels of social anxiety at baseline (mean age 11.0) predicted higher odds of alcohol consumption at 5-year follow-up (Dahne et al., 2014). With regard to the order of onset, the literature largely shows that social anxiety usually precedes problematic alcohol use, including younger age of first 
use and first intoxication, hazardous use, and meeting diagnostic criteria for AUD (Blumenthal et al., 2011; Buckner et al., 2008; Dahne et al., 2014; Marmorstein, 2012; Wolitzky- Taylor et al., 2012; Zimmermann et al., 2003).

The relationship between social anxiety and alcohol use may differ by gender. Compared to males, females have higher rates of social anxiety (Kessler et al., 1994; Xu et al., 2012) and lower rates of AUD (Grant et al., 2004; Hasin et al., 2007). Among adolescents ages 13 to $17, \mathrm{Wu}$ and colleagues (2010) found a negative association between SAD and alcohol use among girls, but no association between SAD and alcohol use among boys. However, gender differences in the relationship of SAD and AUD may vary based on the developmental period studied. Kessler and colleagues (1997) examined a large, nationally representative sample of adolescents and adults (ages 15 to 54) and found that among women, SAD was associated with alcohol abuse and dependence, while SAD was only associated with alcohol dependence among men. Among college students (mean age 19.5), Norberg and colleagues (2009) found that women with high social anxiety, compared to those with low social anxiety, were more likely to experience increased alcohol-related problems per drinking episode, despite drinking similar amounts. Compared to men with high social anxiety, women with high social anxiety were also more likely to experience increased alcohol-related problems (Norberg et al., 2009).

To our knowledge, this is the first study to examine the course of SAD from early adolescence into adulthood and to examine the persistence or desistance of SAD in relationship to AUD symptom course. The first aim was to characterize trajectories of SAD diagnosis and AUD symptoms separately, from ages 12 to 25 . Based on prior research (Grant et al., 2005; Van Oort et al., 2009), we hypothesized that rates of SAD would peak at around age 15 and show a variable course (i.e., remission or persistence). With regard to AUD symptoms, we predicted that there would be multiple trajectories, with at least 1 group showing an onset of AUD symptoms in emerging adulthood (ages 18 to 22) and another group showing severe, persistent AUD symptoms. The second aim of this project was to examine dual trajectories of SAD and AUD symptoms. We hypothesized that among individuals with persisting SAD, compared to those who show remission (i.e., adolescentlimited SAD), there would be an increased probability of membership in a more severe AUD symptom trajectory. The third aim was to test for gender differences in the association of SAD and AUD symptoms over time. We predicted that compared to males with SAD, females with SAD would be more likely to belong to a more severe AUD symptom trajectory group.

\section{MATERIALS AND METHODS}

\section{Participants}

Participants were 788 youth ( $46.2 \%$ female; $80.5 \%$ White; 12 to 18 years old at baseline) who were followed in a longitudinal study at the Pittsburgh Adolescent Alcohol Research Center. Youth were recruited either by random digit dialing to identify a representative community sample $(n=220)$ or from clinical programs that included addictions treatment $(n$ $=568$ ). Details on recruitment procedures and attrition analyses have been published (Clark et al., 2001, 2010). Table 1 summarizes participant characteristics. 


\section{Procedures}

Prior to initiating study procedures, written informed consent or assent was obtained from adolescents; a parent/guardian provided written informed consent for youth under age 18. Participants completed the baseline assessment and were followed at 1,3, and 5 years, and at age 25. At baseline, adolescents completed a day-long assessment protocol to assess substance use, psychiatric diagnoses, and other variables of interest, for example, socioeconomic status. At the baseline assessment, participants retrospectively reported on lifetime SAD and AUD symptoms. During follow-up visits, participants reported on psychiatric symptoms (e.g., SAD and AUD) since their previous assessment. Participants were compensated for their time each visit with a $\$ 125$ gift card. Study procedures were approved by the University of Pittsburgh Human Subjects Institutional Review Board.

\section{Measures}

Demographics-The baseline assessment collected data on gender, race/ethnicity, and socioeconomic status (Hollingshead, 1975).

Alcohol Use Disorder-AUD symptoms and diagnoses were assessed with a modified version of the Structured Clinical Interview for DSM-IV (First et al., 2002; Martin et al., 1995, 2000). Ages of onset and offset for AUD symptoms were used to generate symptom count data for each year from ages 12 to 25 . Highly trained interviewers achieved excellent inter-rater reliabilities for AUD symptom ratings ( $\kappa=0.84$ to 1.00), AUD diagnoses ( $\kappa=$ 0.94), and ages of symptom onset and offset (intraclass $r=0.98$ to 1.00; Martin et al., 2000).

Social Anxiety Disorder-SAD diagnosis was determined using the Kiddie Schedule for Affective Disorders and Schizophrenia- Present and Lifetime version (K-SADS), a widely used semistructured interview for DSM-IV psychiatric disorders in youth and young adults, which also collects information on ages of onset and offset for psychiatric conditions (Kaufman et al., 1997; Puig-Antich et al., 1980). The K-SADS has acceptable to good psychometric properties, with acceptable to high inter-rater reliability for psychiatric diagnoses (mean extended $\kappa$ of 0.88 in the current sample; Clark et al., 1997). Ages of SAD onset and offset were used to determine the presence/absence of SAD for each year from ages 12 to 25 .

\section{Data Analysis}

Multivariate polynomial growth mixture modeling (GMM; Muthén, 2001; Muthén and Shedden, 1999) was performed on SAD and AUD symptoms, separately, as a function of age. SAD (presence or absence) and AUD symptom counts (range: 0 to 11) in the past year were coded at each age, for ages 12 to 25. GMMs were estimated in Mplus using maximumlikelihood estimation with robust standard errors. SAD was treated as a categorical variable using a logit function. AUD symptom counts were treated as count data using a Poisson distribution. All observations were used, with incomplete data handled using full information maximum-likelihood estimation. Models specifying 2 to 6 classes were estimated separately (univariate models) for SAD and AUD symptoms. Random effects (individual variation on intercept and slopes within each class) were tested. Nonsignificant random effects were fixed at 0 for model parsimony. Akaike information criterion (AIC; 
Akaike, 1974), Bayesian information criterion (BIC; Schwarz, 1978), and sample-sizeadjusted BIC (ssBIC; Sclove, 1987) were used to guide model selection. Lower AIC, BIC, and ssBIC values indicate better model fit (Kass and Wasserman, 1995). Model selection was based on consideration of statistical criteria in conjunction with theoretical considerations interpretability of classes (Nagin, 2005). The 2 models selected in the univariate SAD and AUD symptom analyses were entered into a dual trajectory analysis.

Because classification quality was high for each model selected, we used a classify-analyze approach (Roeder et al., 1999) and exported assignment of individuals to the most likely class for chi-square analyses. Chi-square tests were performed to examine gender differences in overall SAD, AUD symptom, and joint SAD/AUD symptom trajectory membership. Then, univariate $z$-tests were performed to examine gender differences by subclasses.

\section{RESULTS}

\section{SAD Trajectory Classes}

A 3-class quadratic logistic GMM for SAD was selected based on model fit indices (see Appendix) along with theoretical considerations and model interpretability. Figure 1 depicts the 3 trajectory classes. Entropy for the selected SAD trajectory model was high (0.98; maximum value $=1.0)$. An adolescent-limited class $(15 \%)$ started with relatively high probability of SAD at ages 12 to 15 , which then decreased through age 25 . The persistent SAD class (6\%) showed increasing probability of SAD between the ages of 14 and 18, and relatively high probability of SAD at ages 19 to 25 . The majority of the sample (79\%) was in the no SAD class.

\section{AUD Symptom Trajectory Classes}

A 5-class quadratic Poisson GMM for AUD symptoms was chosen based on model fit (see Appendix) and interpretability. A plot of the estimated number of AUD symptoms over time for each trajectory class is presented in Fig. 2. Entropy for the selected AUD symptom trajectory model was good (0.78). The stable low class (28\%) generally reported no AUD symptoms at ages 12 to 25 . The remitting class (18\%) had, on average, 2 to 3 symptoms in early adolescence, with a gradual decline in AUD symptom count through age 25 . The young adult onset class (22\%) reported, on average, 1 AUD symptom at ages 23 to 25 . The moderate class (22\%) reported, on average, 2 to 3 symptoms over ages 12 to 25 . The severe class (10\%) reported the highest average number of AUD symptoms, with a peak in AUD symptom count (mean $=5.3)$ at age 20 .

\section{Sex by SAD and AUD symptom trajectory classes}

Sex was significantly associated with SAD trajectory class $\left(\chi^{2}(2, N=788)=32.67\right.$, exact $p$ $<0.001$, Cramér's $V=0.20$ ). Females, compared to males, were more likely to be in the adolescent-limited SAD class ( $16 \%$ vs. $6 \% ; z=2.26, p<0.05)$. There were also more females than males in the persisting SAD class ( $8 \%$ vs. $4 \%$ ), albeit this was not a significant difference $(z=1.16, p=0.23)$. 
There also was a significant relationship between sex and AUD symptom trajectory class $\left(\chi^{2}(4, N=788)=66.44\right.$, exact $p<0.001$, Cramér's $\left.V=0.29\right)$. Overall, males tended to have a worse course of AUD symptoms. Almost one-third (31\%) of males were classified in the moderate AUD symptom class compared to $12 \%$ of females $(z=3.27, p<0.001)$, whereas nearly half of females $(43 \%)$ were classified in the stable low AUD class compared to $20 \%$ of males $(z=3.50, p<0.001)$.

\section{Dual Trajectory Analyses: SAD and AUD Symptom Count}

Entropy for the dual trajectory model was 0.79 (maximum value $=1$ ), indicating good classification quality. Table 2 presents the cross-classification of participants in each AUD class by SAD class in the dual trajectory model, and tests of group differences. In the adolescent-limited SAD class, participants were most commonly classified in AUD stable low (39\%) and AUD remitting (25\%) classes. The distribution of participants in the persisting SAD class across the 5 AUD classes was fairly even. Those in the adolescentlimited SAD class were more likely to be in the stable low AUD class (39\%) compared to those with persistent SAD $(20 \% ; z=2.22, p<0.05)$. Comparison of those with and without $\mathrm{SAD}$ on alcohol trajectories revealed mixed findings. Compared to those with no SAD, those in the adolescent-limited SAD class was more likely to be in the remitting AUD class $(16 \%$ vs. $25 \% ; z=2.23, p<0.05)$ and less likely to be in the young adult onset ( $23 \%$ vs. $12 \% ; z=-2.26, p<0.05)$ and moderate AUD classes (24\% vs. $12 \% ; z=-2.45, p<0.05)$. The proportion of individuals in the severe AUD class was lower among those without SAD (8\%), compared to those with persistent $\operatorname{SAD}(17 \% z=2.34 ; p<0.05)$. Further, there was a lower proportion in the severe AUD class for those with no SAD (8\%), compared to all of those with a history of SAD (combined percentage for the 2 SAD groups $=14 \% ; z=2.36 ; p$ $<0.05)$.

\section{Sex and AUD Symptom Trajectory Classes Among Those with a History of SAD}

Because we were interested in gender differences in alcohol problem trajectories among those with SAD, we tested for differences between males and females after excluding those in the no SAD group, and combining data from the persistent and adolescent-limited SAD groups. Table 3 displays the percentage of males and females with SAD (persistent and adolescent-limited) in each AUD symptom class based on dual trajectory analysis. A 2-way chi-square test of independence was performed on sex and joint AUD/SAD trajectory class, excluding the no SAD class. There was a significant relationship between sex and joint AUD/SAD trajectory class $\chi^{2}(9, N=129)=23.22, p=0.006$, Cramér's $V=0.42$. There was evidence that males with SAD showed greater alcohol problem involvement during early to mid-adolescence compared to females with SAD. Males with SAD were more likely to be in the moderate AUD symptom class than females with SAD (33\% vs. $10 \% ; z=3.13, p<$ $0.01)$. Males with SAD were less likely to be in the stable low class than females with SAD ( $18 \%$ vs. $38 \% ; z=-2.34, p<0.05)$. Males with SAD also were less likely than females with SAD to be in the young adult onset AUD group (5\%vs. 20\%; $z=-2.21 ; p<0.05)$. 


\section{DISCUSSION}

In this longitudinal study of adolescence through young adulthood, we identified 3 trajectories of SAD: no SAD (79\%), adolescent-limited SAD (15\%), and persistent SAD $(6 \%)$. Females were more likely than males to be in the adolescent- limited SAD class. We also identified 5 alcohol symptom trajectory groups: stable low (28\%), remitting (18\%), young adult onset (22\%), moderate (22\%), and severe (10\%). These trajectories are consistent with those found in prior research on alcohol use (Jackson and Sher, 2005). Overall, males, compared to females, belonged to AUD classes characterized by more alcohol symptoms. Dual trajectory analyses revealed that the presence of SAD was associated with the presence of more alcohol symptoms; an absence of a SAD history corresponded with membership in less severe AUD symptom classes. Among those with SAD, there were some gender differences in AUD class membership.

The dual trajectory analyses showed that adolescent-limited SAD and persistent SAD are associated with somewhat different patterns of change in AUD symptoms from adolescence into young adulthood. A greater proportion of individuals in the adolescent-limited SAD class belonged to the stable low AUD symptom group compared to the persisting SAD class (39\% vs. 20\%). These results suggest that the persistence of SAD into young adulthood may have some functional associations with alcohol problems.

Social anxiety typically precedes the onset of problematic alcohol use (Kessler et al., 2005b; Tomlinson and Brown, 2012; Wolitzky-Taylor et al., 2012) which has led researchers to theorize that SAD contributes to the onset of AUD symptoms (Buckner et al., 2013; Kushner et al., 2000). The current study identified multiple patterns in order of onset of SAD and alcohol symptoms. Specifically, adolescent-limited SAD preceded young adult onset AUD in some youth, but the combination of remitting AUD and persistent SAD (i.e., alcohol symptoms prior to SAD onset) also was observed. The most common combinations that were identified in this sample were adolescent-limited SAD and stable low AUD, and adolescent-limited SAD and remitting AUD. The heterogeneity in patterns of SAD and AUD from adolescence into young adulthood suggests specific patient subgroups that may respond to tailored interventions. For example, treatment of SAD that onsets during adolescence may mitigate subsequent alcohol problems.

Examination of gender and AUD symptom trajectories among participants with SAD revealed mixed and unexpected findings. Compared to females with SAD, males with SAD were less likely to be in the stable low AUD class and more likely to be in the moderate AUD class. Compared to females with SAD, males with SAD also were less likely to have a young adult onset of AUD symptoms. The data are consistent with the idea that both genders show positive associations between SAD with alcohol symptoms, but males with SAD are more likely to have alcohol problems in early to mid-adolescence, whereas females with SAD are more likely to have alcohol problems later adolescence and young adulthood. More research is needed to replicate and extend these findings.

With regard to development, the current findings suggest that for females, social anxiety and alcohol problems may be associated in young adulthood. Data indicated that females with 
SAD were more likely than males with SAD to be in the young adult onset AUD class. Some existing cross-sectional research on young adults has also found that young adult females with high levels of social anxiety, compared to similar males, have more alcoholrelated problems (Norberg et al., 2009). In a large community sample of younger youth (ages 13 to 17), SAD was protective against all substance use for girls (Wu et al., 2010). The current study extends the age range covered by Wu and colleagues (2010) and suggests that age 18 might mark an important turning point for some females with SAD in risk for alcohol symptoms. The current results suggest that gender differences in the association of SAD and AUD symptoms may be specific to the developmental period studied. It is also important to note that $\mathrm{Wu}$ and colleagues (2010) examined substance use, not substance use-related problems, which could potentially affect gender findings.

More research is needed to understand gender differences in SAD and its association with alcohol problems. While not examined in the current study, type of alcohol problems and the severity of social anxiety may be important factors to examine when dissecting the role of gender in the relation to co-occurring social anxiety and AUD symptoms or consequences (Norberg et al., 2011). Research should examine whether gender differences are explained in part by common cultural stereotypes of masculinity, which may lead males to drink earlier in adolescence (Addis and Mahalik, 2003). It is possible that males with SAD might learn early to drink in social contexts in an effort to mask symptoms of SAD and enhance positive aspects of social experiences.

Study limitations warrant comment. Generalizability may be limited, given the parent study's sampling strategy — as a clinical group was recruited to produce a sample with a high proportion of AUDs. Other issues pertinent to generalizability are that the majority of our sample was White and some combinations of SAD and AUD trajectory classes had a small number of participants. It is important to keep in mind that growth mixture models provide estimates of trajectory classes which warrant replication in other samples. Correlates of the trajectory classes other than gender were not examined, given the complexity of interpreting results involving multiple covariates in dual trajectory models. Future projects may benefit from analysis of SAD symptoms, in addition to the SAD diagnoses studied here, as this may improve sensitivity to detecting change in severity of social anxiety and its relation to alcohol problems over time. Another fruitful direction may be to examine the type of alcohol problems reported among those with persisting SAD, as this could improve the understanding of the relationship of SAD and AUD, and advance treatment efforts.

Future research should examine mechanisms explaining the relationship between SAD and AUD symptoms. Buckner and colleagues (2013) recently proposed a biopsychosocial model of SAD and substance use, which provides a more thorough conceptual framework than a stress-dampening model. Guided by Buckner and colleagues' model (2013), alcohol use among those with social anxiety may reflect a coping strategy to manage negative evaluation fears, increase positive affect, and facilitate socialization. Research is needed to test such potential mechanisms linking SAD and AUD.

In summary, study findings suggest 3 SAD classes: no SAD, adolescent-limited, and persisting. Each SAD trajectory class was associated with distinct patterns of change in 
AUD symptoms from adolescence into young adulthood. Developmental period affected gender differences in the relationship of SAD and AUD. Findings suggest that treatment for $\mathrm{SAD}$ in adolescence may mitigate subsequent alcohol problems.

\section{Acknowledgments}

This research project was funded by NIAAA T32 AA07453 (JJB), R01AA016482 (DBC), P50DA05605 (DBC), 5R01AA013397 and K24 AA020840 (CSM), and K02 AA018195 (TC).

\section{References}

Addis ME, Mahalik JR. Men, masculinity, and the contexts of help seeking. Am Psychol. 2003; 58:514. [PubMed: 12674814]

Akaike H. A new look at the statistical model identification. IEEE Trans Automat Contr. 1974; 19:716-723.

American Psychiatric Association. Diagnostic and Statistical Manual of Mental Disorders. 5. American Psychiatric Association; Washington, DC: 2013.

Blumenthal H, Leen-Feldner EW, Badour CL, Babson KA. Anxiety psychopathology and alcohol use among adolescents: a critical review of the empirical literature and recommendations for future research. J Exp Psychopathol. 2011; 2:318-353. [PubMed: 23243493]

Buckner JD, Heimberg RG, Ecker AH, Vinci C. A biopsychosocial model of social anxiety and substance use. Depress Anxiety. 2013; 30:276-284. [PubMed: 23239365]

Buckner JD, Schmidt NB, Lang AR, Small JW, Schlauch RC, Lewinsohn PM. Specificity of social anxiety disorder as a risk factor for alcohol and cannabis dependence. J Psychiatr Res. 2008; 42:230-239. [PubMed: 17320907]

Chassin L, Fora DB, King KM. Trajectories of alcohol and drug use and dependence from adolescence to adulthood: the effects of familial alcoholism and personality. J Abnorm Psychol. 2004; 113:483498. [PubMed: 15535782]

Chung, T.; Jackson, K. Adolescent alcohol use. In: Brown, S.; Zucker, R., editors. Oxford Handbook of Adolescent Substance Use. Oxford; New York, NY: in press

Chung, T.; Martin, CS. Adolescent substance use and substance use disorders: prevalence and clinical course. In: Kaminer, Y.; Winters, K., editors. Clinical Manual of Adolescent Substance Use Treatment. American Psychiatric Press; Washington, DC: 2011. p. 1-24.

Clark DB, Lynch KG, Donovan JE, Block GD. Health problems in adolescents with alcohol use disorders: self-report, liver injury, and physical examination findings and correlates. Alcohol Clin Exp Res. 2001; 25:1350-1359. [PubMed: 11584156]

Clark DB, Pollock N, Bukstein OG, Mezzich AC, Bromberger JT, Donovan JE. Gender and comorbid psychopathology in adolescents with alcohol dependence. J Am Acad Child Adolesc Psychiatry. 1997; 36:1195-1203. [PubMed: 9291720]

Clark DB, Thatcher DL, Martin CS. Child abuse and other traumatic experiences, alcohol use disorders, and health problems in adolescence and young adulthood. J Pediatr Psychol. 2010; 35:499-510. [PubMed: 19966317]

Dahne J, Banducci AN, Kurdziel G, Macpherson L. Early adolescent symptoms of social phobia prospectively predict alcohol use. J Stud Alcohol Drugs. 2014; 75:929-936. [PubMed: 25343649]

First, MB.; Spitzer, RL.; Gibbon, M.; Williams, JBW. Biometrics Research. New York State Psychiatric Institute; New York, NY: 2002. Structured Clinical Interview for DSM-IV-TR Axis I Disorders, Research Version, Non- Patient Edition (SCID-I/NP).

Grant BF, Dawson DA, Stinson FS, Chou SP, Dufour MC, Pickering RP. The 12-month prevalence and trends in DSM-IV alcohol abuse and dependence: United States, 1991-1992 and 2001-2002. Drug Alcohol Depend. 2004; 74:223-234. [PubMed: 15194200]

Grant BF, Hasin DS, Blanco C, Stinson FS, Chou SP, Goldstein RB, Dawson DA, Smith S, Saha TD, Huang B. The epidemiology of social anxiety disorder in the United States: results from the 
National Epidemiologic Survey on Alcohol and Related Conditions. J Clin Psychiatry. 2005; 66:1351-1361. [PubMed: 16420070]

Hasin DS, Stinson FS, Ogburn E, Grant BF. Prevalence, correlates, disability, and comorbidity of DSM-IV alcohol abuse and dependence in the United States: results from the National Epidemiologic Survey on Alcohol and Related Conditions. Arch Gen Psychiatry. 2007; 64:830 842. [PubMed: 17606817]

Hollingshead, A. Two-Factor Index of Social Status. Yale University; NewHaven, CT: 1975.

Jackson KM, Sher KJ. Similarities and differences of longitudinal phenotypes across alternate indices of alcohol involvement: a methodologic comparison of trajectory approaches. Psychol Addict Behav. 2005; 19:339-351. [PubMed: 16366806]

Kass RE, Wasserman L. A reference Bayesian test for nested hypotheses and its relationship to the Schwarz criterion. J Am Stat Assoc. 1995; 90:928-934.

Kaufman J, Birmaher B, Brent D, Rao U, Flynn C, Moreci P, Williamson D, Ryan N. Schedule for Affective Disorders and Schizophrenia for School-Age Children-Present and Lifetime Version (KSADS-PL): initial reliability and validity data. J Am Acad Child Adolesc Psychiatry. 1997; 36:980-988. [PubMed: 9204677]

Kessler RC, Adler L, Ames M, Barkley RA, Birnbaum H, Greenberg P, Johnston JA, Spencer T, Ustun TB. The prevalence and effects of adult attention deficit/hyperactivity disorder on work performance in a nationally representative sample of workers. J Occup Environ Med. 2005a; 47:565-572. [PubMed: 15951716]

Kessler RC, Angermeyer M, Anthony JC, De Graaf R, Demyttenaere K, Gasquet I, De Girolamo G, Gluzman S, Gureje O, Haro JM, Kawakami N, Karam A, Levinson D, Medina Mora ME, Oakley Browne MA, Posada-Villa J, Stein DJ, Adley Tsang CH, Aguilar-Gaxiola S, Alonso J, Lee S, Heeringa S, Pennell BE, Berglund P, Gruber MJ, Petukhova M, Chatterji S, Ustün TB. Lifetime prevalence and age-of-onset distributions of mental disorders in the World Health Organization's World Mental Health Survey Initiative. World Psychiatry. 2007; 6:168-176. [PubMed: 18188442]

Kessler RC, Berglund P, Demler O, Jin R, Merikangas KR, Walters EE. Lifetime prevalence and ageof-onset distributions of DSM-IV disorders in the National Comorbidity Survey Replication. Arch Gen Psychiatry. 2005b; 62:593-602. [PubMed: 15939837]

Kessler RC, Crum RM, Warner LA, Nelson CB, Schulenberg J, Anthony JC. Lifetime co-occurrence of DSM-III-R alcohol abuse and dependence with other psychiatric disorders in the National Comorbidity Survey. Arch Gen Psychiatry. 1997; 54:313-321. [PubMed: 9107147]

Kessler RC, McGonagle KA, Zhao S, Nelson CB, Hughes M, Eshleman S, Wittchen HU, Kendler KS. Lifetime and 12-month prevalence of DSM-III-R psychiatric disorders in the United States. Results from the National Comorbidity Survey. Arch Gen Psychiatry. 1994; 51:8-19. [PubMed: 8279933]

Kushner MG, Abrams K, Borchardt C. The relationship between anxiety disorders and alcohol use disorders: a review of major perspectives and findings. Clin Psychol Rev. 2000; 20:149-171. [PubMed: 10721495]

Marmorstein NR. Anxiety disorders and substance use disorders: different associations by anxiety disorder. J Anxiety Disord. 2012; 26:88-94. [PubMed: 22018969]

Martin CS, Kaczynski NA, Maisto SA, Bukstein OM, Moss HB. Patterns of DSM-IV alcohol abuse and dependence symptoms in adolescent drinkers. J Stud Alcohol. 1995; 56:672-680. [PubMed: 8558899]

Martin CS, Pollock NK, Bukstein OG, Lynch KG. Inter-rater reliability of the SCID alcohol and substance use disorders section among adolescents. Drug Alcohol Depend. 2000; 59:173-176. [PubMed: 10891630]

Muthén, B. Latent variable mixture modeling. In: Marcoulides, GA.; Schumacker, RE., editors. New Developments and Techniques in Structural Equation Modeling. Lawrence Erlbaum Associates; Mahwah, NJ: 2001. p. 1-34.

Muthén B, Shedden K. Finite mixture modeling with mixture outcomes using the EM algorithm. Biometrics. 1999; 55:463-469. [PubMed: 11318201]

Nagin, DS. Group-Based Modeling of Development. Harvard University Press; Cambridge, MA: 2005. 
Norberg MM, Norton AR, Olivier J. Refining measurement in the study of social anxiety and student drinking: who you are and why you drink determines your outcomes. Psychol Addict Behav. 2009; 23:586-597. [PubMed: 20025365]

Norberg MM, Olivier J, Alperstein DM, Zvolensky MJ, Norton AR. Adverse consequences of student drinking: the role of sex, social anxiety, drinking motives. Addict Behav. 2011; 36:821-828. [PubMed: 21481541]

Puig-Antich, J.; Tabrizi Ma, OH.; Chambers, W. The Schedule for Affective Disorders and Schizophrenia for School-Aged Children-Epidemiologic Version. New York State Psychiatric Institution and Yale University School of Medicine; New York, NY: 1980.

Roeder K, Lynch KG, Nagin DS. Modeling uncertainty in latent class membership: a case study in criminology. J Am Stat Assoc. 1999; 94:766-776.

Schwarz G. Estimating the dimension of the model. Ann Stat. 1978; 6:461-464.

Sclove S. Application of the model-selection criteria to some problems in multivariate analysis. Psychometrika. 1987; 52:333-343.

Tomlinson KL, Brown SA. Self-medication or social learning? A comparison of models to predict early adolescent drinking. Addict Behav. 2012; 37:179-186. [PubMed: 22055793]

Van Oort FV, Greaves-Lord K, Verhulst FC, Ormel J, Huizink AC. The developmental course of anxiety symptoms during adolescence: the TRAILS study. J Child Psychol Psychiatry. 2009; 50:1209-1217. [PubMed: 19490305]

Windle M, Windle RC. Testing the specificity between social anxiety disorder and drinking motives. Addict Behav. 2012; 37:1003-1008. [PubMed: 22626889]

Wolitzky-Taylor K, Bobova L, Zinbarg RE, Mineka S, Craske MG. Longitudinal investigation of the impact of anxiety and mood disorders in adolescence on subsequent substance use disorder onset and vice versa. Addict Behav. 2012; 37:982-985. [PubMed: 22503436]

Wu P, Goodwin RD, Fuller C, Liu X, Comer JS, Cohen P, Hoven CW. The relationship between anxiety disorders and substance use among adolescents in the community: specificity and gender differences. J Youth Adolesc. 2010; 39:177-188. [PubMed: 20084563]

Xu Y, Schneier F, Heimberg RG, Princisvalle K, Liebowitz MR, Wang S, Blanco C. Gender differences in social anxiety disorder: results from the national epidemiologic sample on alcohol and related conditions. J Anxiety Disord. 2012; 26:12-19. [PubMed: 21903358]

Zimmermann P, Wittchen HU, Hofler M, Pfister H, Kessler RC, Lieb R. Primary anxiety disorders and the development of subsequent alcohol use disorders: a 4-year community study of adolescents and young adults. Psychol Med. 2003; 33:1211-1222. [PubMed: 14580076]

\section{APPENDIX}

\section{Table A1}

Information Criteria for Multi-Class Logistic Regression Models of Social Anxiety Disorder by Age

\begin{tabular}{lcccc}
\hline \# Classes & LogL & AIC & BIC & ssBIC \\
\hline 2 & -1483.87 & 2981.73 & 3014.42 & 2992.19 \\
3 & -1282.90 & 2587.79 & 2639.16 & 2604.23 \\
4 & -1198.34 & 2426.68 & 2496.72 & 2449.09 \\
5 & -1151.67 & 2341.34 & 2430.06 & 2369.73 \\
6 & -1139.45 & 2324.91 & 2432.31 & 2359.27 \\
\hline
\end{tabular}

AIC, Akaike information criterion; BIC, Bayesian information criterion; ssBIC, sample-size-adjusted BIC. 


\section{Table A2}

Information Criteria for Multi-Class Poisson Regression Models of Alcohol Use Disorder Symptoms by Age

\begin{tabular}{lcccc}
\hline \# Classes & LogL & AIC & BIC & ssBIC \\
\hline 2 & -15621.51 & 31257.02 & 31289.67 & 31267.44 \\
3 & -14727.51 & 29477.03 & 29528.33 & 29493.40 \\
4 & -13927.59 & 27885.18 & 27955.14 & 27907.51 \\
5 & -13562.97 & 27163.93 & 27252.56 & 27192.22 \\
6 & -13307.57 & 26661.14 & 26768.42 & 26695.38 \\
\hline
\end{tabular}

AIC, Akaike information criterion; BIC, Bayesian information criterion; ssBIC, sample-size-adjusted BIC. 


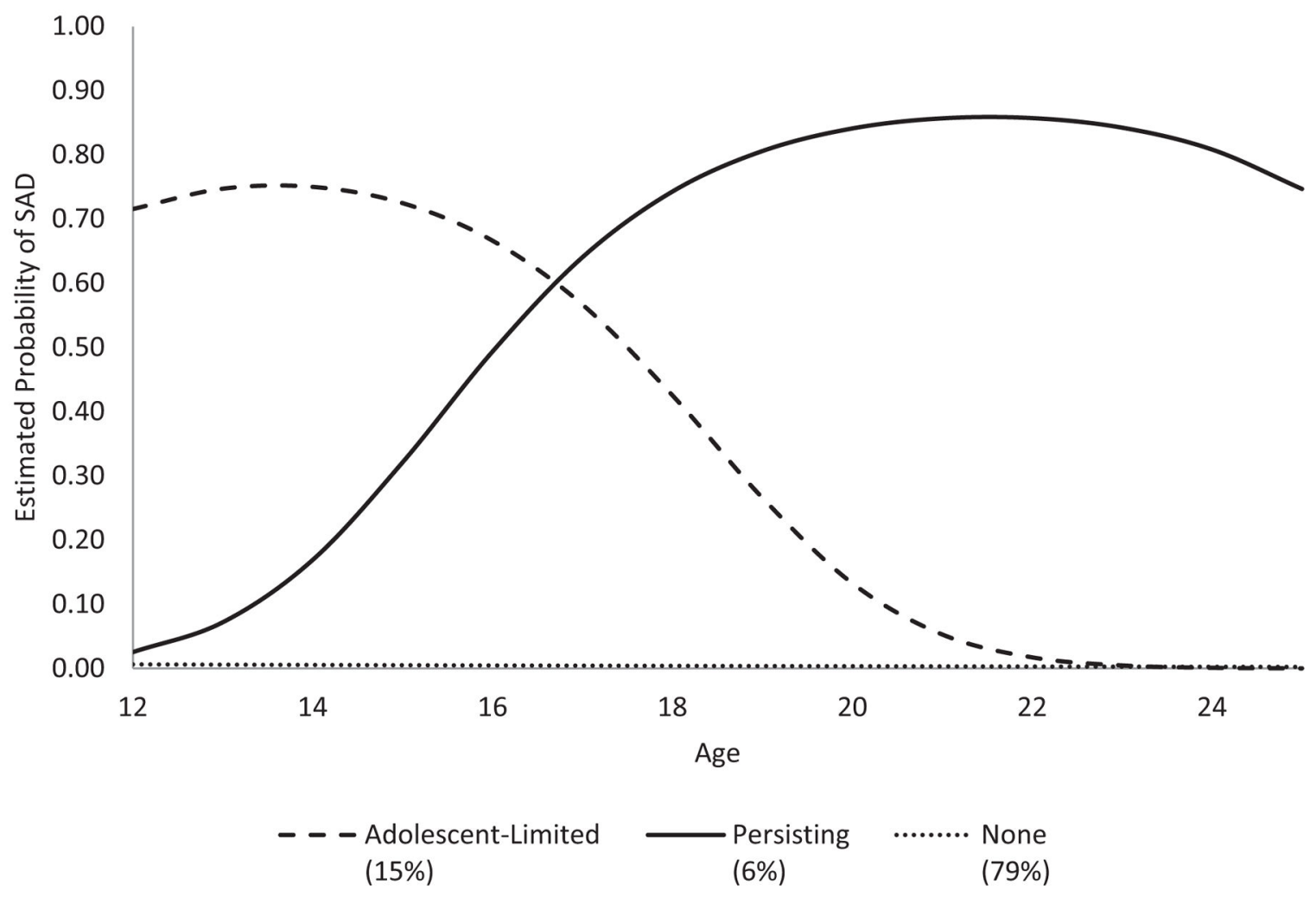

Fig. 1.

Predicted probability of social anxiety disorder (SAD) by age for each SAD trajectory class. 


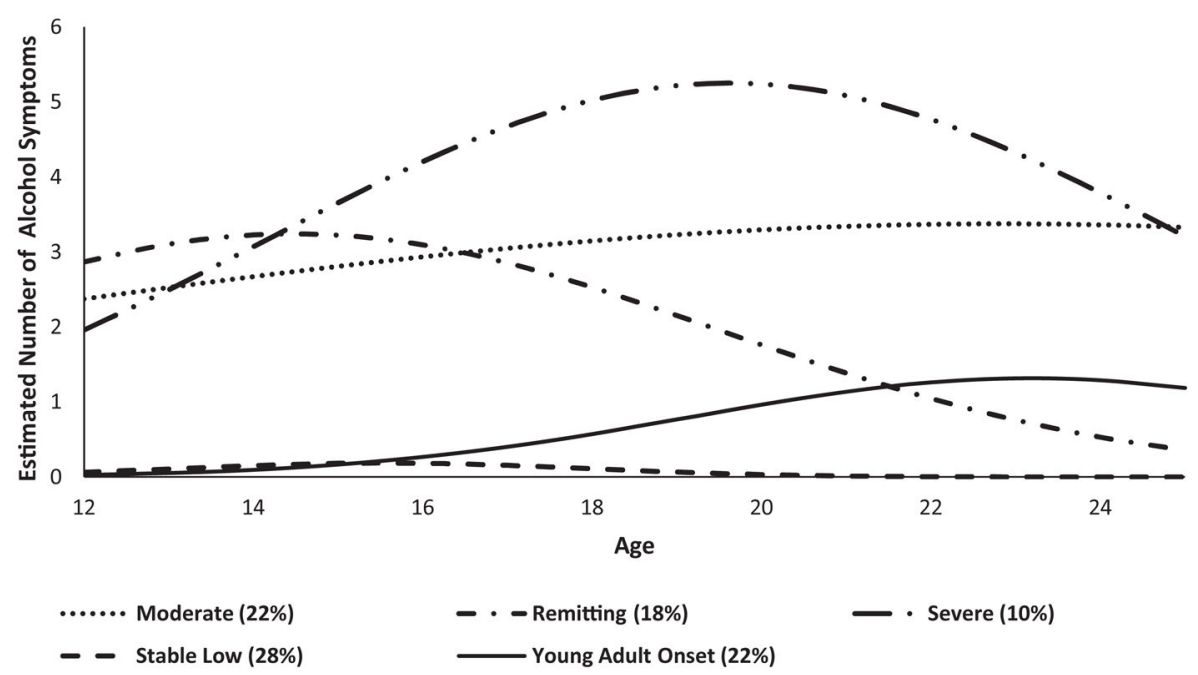

Fig. 2.

Predicted count of alcohol use disorder (AUD) symptoms by age for each AUD symptom trajectory class. 


\section{Table 1}

Sample Characteristics $(N=788)$

\begin{tabular}{|c|c|c|c|}
\hline & $\begin{array}{c}\text { Total } N=788 \%(N) \text { or } \\
\text { Mean (SD) }\end{array}$ & $\begin{array}{c}\text { Community } n=220 \%(N) \\
\text { or Mean (SD) }\end{array}$ & $\begin{array}{c}\text { Clinical } n=568 \%(N) \text { or } \\
\text { Mean (SD) }\end{array}$ \\
\hline Female & $46.2 \%(364)$ & $52.7 \%(116)$ & $43.7 \%(248)$ \\
\hline Race: White & $80.5 \%(634)$ & $81.4 \%(179)$ & $80.1 \%(455)$ \\
\hline Black & $18.8 \%(148)$ & $17.7 \%(39)$ & $19.2 \%(109)$ \\
\hline Other race & $0.07 \%(6)$ & $0.09 \%(2)$ & $0.07 \%(4)$ \\
\hline SES & $2.78(1.14)$ & $2.29(0.98)$ & $2.97(1.15)$ \\
\hline \multicolumn{4}{|l|}{ Alcohol symptom count } \\
\hline Age $12 ; n=760$ & $0.19(0.84)$ & $0.01(0.10)$ & $0.26(0.99)$ \\
\hline Age $14 ; n=760$ & $1.08(1.97)$ & $0.10(0.45)$ & $1.47(2.21)$ \\
\hline Age $16 ; n=756$ & $1.98(2.69)$ & $0.31(1.01)$ & $2.65(2.86)$ \\
\hline Age $18 ; n=722$ & $1.58(2.31)$ & $0.49(1.19)$ & $2.04(2.50)$ \\
\hline Age $20 ; n=677$ & $1.45(2.01)$ & $0.83(1.63)$ & $1.72(2.22)$ \\
\hline Age $22 ; n=634$ & $1.45(2.01)$ & $0.87(1.52)$ & $1.72(2.15)$ \\
\hline Age $24 ; n=593$ & $1.47(2.16)$ & $0.70(1.44)$ & $1.80(2.33)$ \\
\hline Age $25 ; n=514$ & $1.48(2.14)$ & $0.61(1.26)$ & $1.86(2.33)$ \\
\hline \multicolumn{4}{|l|}{ Social anxiety disorder } \\
\hline Age $12 ; n=788$ & $6.5 \%(51)$ & $4.1 \%(9)$ & $7.4 \%(42)$ \\
\hline Age $14 ; n=787$ & $8.9 \%(70)$ & $4.1 \%(9)$ & $10.8 \%(61)$ \\
\hline Age $16 ; n=771$ & $9.6 \%(74)$ & $5.1 \%(11)$ & $11.4 \%(63)$ \\
\hline Age $18 ; n=722$ & $7.6 \%(55)$ & $4.3 \%(9)$ & $9.0 \%(46)$ \\
\hline Age $20 ; n=677$ & $6.5 \%(44)$ & $2.9 \%(6)$ & $8.1 \%(38)$ \\
\hline Age $22 ; n=634$ & $6.3 \%(40)$ & $2.0 \%(4)$ & $8.3 \%(36)$ \\
\hline Age $24 ; n=593$ & $5.4 \%(32)$ & $1.7 \%(3)$ & $7.0 \%(29)$ \\
\hline Age $25 ; n=514$ & $4.9 \%(25)$ & $1.3 \%(2)$ & $6.4 \%(23)$ \\
\hline Baseline \# of drinking occasions/month ${ }^{*}$ & $6.4(9.3)$ & $1.3(2.6)$ & $8.5(10.3)$ \\
\hline Baseline average drinking quantity per occasion ${ }^{*}$ & $5.9(5.7)$ & $2.2(2.9)$ & $7.5(5.8)$ \\
\hline Baseline alcohol use diagnosis (lifetime) & $53.3 \%(420)$ & $8.2 \%(18)$ & $70.8 \%(402)$ \\
\hline Alcohol abuse & $23.9 \%(188)$ & $5.5 \%(12)$ & $31.0 \%(176)$ \\
\hline Alcohol dependence & $29.4 \%(232)$ & $2.7 \%(6)$ & $39.8 \%(226)$ \\
\hline
\end{tabular}

SES, socioeconomic status ( 1 = high SES and 5 = low SES); alcohol symptom count, count of DSM-IV alcohol abuse and dependence symptoms at a given age; social anxiety disorder, met DSM-IV social anxiety disorder criteria at a given age.

$*$

$N=747$ to 748 due to missing data. Only data from selected ages are presented; complete data at each age from ages 12 to 25 are available. 
Table 2

Cross-Classification of SAD and AUD Symptom Class Membership

\begin{tabular}{lcccc}
\hline \multicolumn{5}{c}{ SAD class } \\
\hline AUD class & Adolescent-limited $\boldsymbol{n}(\boldsymbol{\%})$ & Persistent $\boldsymbol{n}(\boldsymbol{\%})$ & No SAD $\boldsymbol{n}(\boldsymbol{\%})$ & Group differences $\boldsymbol{p}<\mathbf{0 . 0 5}$ \\
\hline Stable low & $32(39)$ & $9(20)$ & $197(30)$ & $\mathrm{A}$ \\
Remitting & $21(25)$ & $10(22)$ & $103(16)$ & B \\
Young adult onset & $10(12)$ & $11(24)$ & $151(23)$ & B \\
Moderate & $10(12)$ & $8(17)$ & $158(24)$ & B \\
Severe & $10(12)$ & $8(17)$ & $50(8)$ & C \\
Total & $83(100)$ & $46(100)$ & $659(100)$ & \\
\hline
\end{tabular}

"A" indicates a difference between the adolescent-limited and persistent SAD groups. "B" indicates a difference between the adolescent-limited SAD and no SAD groups. "C" indicates a difference between the persistent SAD and no SAD groups. AUD, alcohol use disorder; SAD, social anxiety disorder. 
Table 3

Proportion of Males and Females with a History of SAD in each AUD Trajectory Class

\begin{tabular}{lccc}
\hline & Males with SAD $n(\%)$ & Females with SAD $n(\%)$ & $z$-Score \\
\hline AUD class & & & \\
Stable low & $7(18)$ & $34(38)$ & $-2.34^{*}$ \\
Remitting & $13(33)$ & $16(18)$ & 1.83 \\
Young adult onset & $2(5)$ & $18(20)$ & $-2.21^{*}$ \\
Moderate & $13(33)$ & $9(10)$ & $3.13^{* *}$ \\
Severe & $5(13)$ & $12(13)$ & -0.15 \\
Total & $n=40(100)$ & $n=89(100)$ & \\
\hline
\end{tabular}

* Significant at $<0.05$;

** significant at $<0.01$.

AUD, alcohol use disorder; SAD, social anxiety disorder. 